ARTÍCULO CORTO DE INVESTIGACIÓN CIENTÍFICA

DOI: 10.47864/SE(50)2020p13-25_114
Sociedad Colombiana
de la Ciencia del Suelo

\title{
SILICIO DISPONIBLE EN SUELOS ALCALINOS POR DIFERENTES MÉTODOS Y EL ABSORBIDO POR CULTIVOS DE MAIZ Y FRIJOL A NIVEL DE INVERNADERO
}

\author{
Enrique Combatt Caballero ${ }^{l} \bowtie$, Daniel A. Palacio Badel ${ }^{2}$, Ruben Blanco Fuentes ${ }^{3}$
}

1 Ph.D. Suelos y nutrición de plantas.
Universidad de Córdoba, Montería-
ORCID:
http://orcid.org/0000-0002-8760-0089
emcombatt@correo.unicordoba.edu.c
o
2 Ph.D en química. Faculty of
Chemistry, University of Concepcion,
Chile, ORCID: orcid.org/0000-0003-
$1874-9696$
3 Facultad de Ciencias Agrícolas,
Universidad de Córdoba, Montería,
Colombia.
https://orcid.org/0000-0002-4423-
4912
Palabras Claves:
mineral,silicio, cultivos,
metodologías analíticas,
elemento químico, nutrición

\section{RESUMEN}

El silicio (Si) es un elemento benéfico, debido que al asociarse con los constituyentes de la pared celular los hace menos susceptibles al ataque de patógenos. El objetivo fue comparar el silicio disponible extraído con cuatro extractores químicos en suelos alcalinos y su correlación con el absorbido por los cultivos de maíz y frijol caupí. El experimento fue realizado en la Facultad de Ciencias Agrícolas de la Universidad de Córdoba y se utilizó un diseño completamente aleatorizado con arreglo factorial, $2 \times 3$ (dos cultivos, tres suelos alcalino) y cuatro repeticiones. Los métodos de extracción fueron: cloruro de calcio 0,01 mol L-1, ácido acético $0,5 \mathrm{~mol} \mathrm{~L}-1$, acetato de amonio $38,5 \mathrm{~g} \mathrm{~L}-1$ y el método propuesto por los autores denominado agua modificada (Agua más boro). Las variables evaluadas fueron el contenido de Si extraído del suelo por diferentes métodos y el Si que se encuentra en la parte vegetativa de cada cultivo y los datos fueron analizados mediante análisis de variancia y correlación. Los resultados indican que el método más eficaz para extraer silicio en de suelos alcalinos para el cultivo de frijol y maíz fue el método de ácido acético 0,5 mol L-1 y el que menos extrajo fue el cloruro de calcio 0,01 mol L-1. Además, el CaCl2 0,01 mol L-1 y el método propuesto por los autores puede ser una alternativa de uso en la estimación de silicio disponible en suelos alcalinos. El silicio absorbido por los cultivos y el extraído por los métodos depende directamente de la especie indicadora y del extractor utilizado.

\section{SILICON AVAILABLE IN ALKALINE SOILS BY DIFFERENT METHODS AND ABSORBED BY CORN AND BEAN CROPS AT GREENHOUSE LEVEL}

\section{Keywords:}

silicon, cultures, analytical methodologies, chemical element, mineral nutrition.

\begin{abstract}
Silicon (Si) is a beneficial element for different crops that are currently being studied. The objective was to evaluate the available silicon extracted with four chemical extractors in alkaline soils and its correlation with that extracted by maize and cowpea bean crops. The experiment was carried out at the Faculty of Agricultural Sciences of the University of Córdoba. A completely randomized design with factorial arrangement, $2 \times 3 \times 4$ (two cultures, three alkaline soils and four repetitions) was used. The extraction methods were: calcium chloride $(0.01 \mathrm{~mol}$ L-1), acetic acid ( $0.5 \mathrm{~mol}$ L-1), ammonium acetate to $38.5 \mathrm{~g} \mathrm{L-1}$ and the method proposed by the authors called modified water. The variables evaluated were the content of Si extracted from the soil by different methods and the Si in the leaves. Quantification was performed in a spectrophotometer at $660 \mathrm{~nm}$ and the data were analyzed with analysis of variance and correlation. The results indicate that the most effective method to extract silicon from alkaline soils for the cultivation of beans and corn was the $0.5 \mathrm{~mol} L-1$ acetic acid method and the one that extracted the least was $0.01 \mathrm{~mol}$ L-1 calcium chloride. Furthermore, CaCl2 $0.01 \mathrm{~mol} \mathrm{L-1}$ and the method proposed by the authors may be an alternative for use in estimating available silicon in alkaline soils. The silicon absorbed by the cultures and the one extracted by the methods depends directly on the indicator species and the extractor used..
\end{abstract}




\section{INTRODUCCIÓN}

El silicio ( $\mathrm{Si}$ ) es un elemento químico poco conocido en la agricultura y su extracción y cuantificación se han estudiado muy poco y específicamente en suelos alcalinos no se ha estudiado. Debido a esto, se ha obstaculizado a los investigadores y agricultores, a estudiar y conocer el beneficio de este elemento en la nutrición mineral de las plantas (Inocencio et al., 2010). De acuerdo con Sandim et al. (2010) y Huang et al. (2011) el silicio se considera un nutriente vegetal benéfico que se acumula en las hojas de algunas gramíneas, formando una barrera protectora contra el ataque de insectos y hongos, regula la pérdida de agua por la planta y la evapotranspiración, aumenta la productividad y calidad y ofrece a una amplia variedad de cultivos, una mayor resistencia al estrés biótico y abiótico (Guntzer et al., 2012; Haynes, 2014; Tubana et al., 2016; Crooks y Prentice, 2017).

En Colombia son pocos los laboratorios que ofrecen el servicio de cuantificación de este elemento, no obstante, muchos agricultores lo utilizan para buscar mayores rendimientos y calidad de las cosechas. Por lo tanto, si las metodologías químicas no están calibradas y correlacionadas con el Si absorbido por los cultivos, no se podrá cuantificar el contenido real que presentan los suelos y su necesidad de aplicación o no para los cultivos de interés en cada zona productora en nuestro país. Según Tubana et al. (2016) y Haynes (2017) existe abundante literatura que indica que el $\mathrm{Si}$ es favorable para los cultivos, pero la cuantificación de la biodisponibilidad sigue es un tema desafiante (Haynes, 2014) y existe la necesidad de buscar métodos que permitan determinar en forma confiable las concentraciones de los elementos nutricionales del suelo.
Según Combatt et al. (2017) eexisten diferentes métodos para predecir los nutrientes que son absorbidos por los cultivos a partir del contenido presente en los suelos y la mayoría de ellos utilizan el reemplazamiento del elemento a extraer por otro de igual característica aniónicas o catiónicas, pero muchas veces sucede el desplazamiento de la forma intercambiable o disponible sin conocer las reacciones químicas que se presentan. Sauer et al. (2006) aplicaron un anión para reemplazar el $\mathrm{Si}$ adsorbido $\mathrm{y}$ han determinado las correlaciones entre el $\mathrm{Si}$ analizado en el extracto y el rendimiento del cultivo.

Actualmente las soluciones extractoras más utilizadas para evaluar la disponibilidad de silicio para las plantas son el acetato de amonio 38,5 $\mathrm{g} \mathrm{L}^{-1}$ (Pereira et al., 2007), ácido acético $0,5 \mathrm{~mol} \mathrm{~L}^{-1}$ y el cloruro de calcio 0,01 mol L-1 (Korndörfer et al., 2004). Sin embargo, los extractantes más exitosos son las soluciones ácidas en lugar de las neutras, gracias a la liberación de una mayor cantidad de protones debido al carácter acido del extractor, pero en algunos casos, estos tienden a sobreestimar los niveles reales de Si disponible en el suelo (Meunier et al., 2018; Crusciol et al., 2018). De acuerdo Patil et al. (2017) la disolución de Si se incrementa aún más por los agentes quelantes, debido a la disminución de la sorción de $\mathrm{Si}$ resultante de la menor concentración de $\mathrm{Al}$ y Fe en la solución.

Otros factores (Liang et al., 2015b; Patil et $a l ., 2017)$ como el método de equilibrio, la relación suelo: solución, la temperatura y pH de la solución extractora, así como la textura, conductividad eléctrica, materia orgánica, iones acompañantes del suelo, entre otros, también son importantes y se deben tener en cuenta en la evaluación de la 
biodisponibilidad de Si. A pesar de anterior, la cantidad de Si varía dependiendo del método de extracción utilizado para solubilizar el Si del suelo (Haynes, 2014; Patil et al., 2017).

Según lo indican Combatt et al. (2017), en Colombia, son pocos los estudios realizados para determinar la concentración de $\mathrm{Si}$ disponible en los suelos, por consiguiente, su aplicación como nutriente esencial, está supeditada a la apreciación del productor o del asistente técnico y muchas veces hay necesidad de aplicarlo o no, dependiendo de la evolución de los suelos. Así mismo, Babu et al. (2016), denotan que identificar el método de extracción más apropiado es otro aspecto esencial a tener en cuenta al recomendar fertilizantes, pues la predicción de las respuestas de los cultivos a la aplicación de Si requiere la calibración del estado del Si del suelo y la absorción de la planta.Por lo tanto, el objetivo de esta investigación fue evaluar el silicio disponible en suelos alcalinos por medio de cuatro extractores químicos y su correlación con el absorbido por los cultivos de maíz (Zea mays L.) y frijol (Vigna unguiculata L. Walp) utilizados como especies indicadoras.

\section{MATERIALES Y MÉTODOS}

El estudio fue realizado en el laboratorio de Suelos y Aguas de la Facultad de Ciencias Agrícolas de la Universidad de Córdoba. En total se colectaron tres suelos con características alcalinas provenientes de los municipios de Moñitos (Córdoba), Planeta Rica (Córdoba) y Sincelejo (Sucre), siendo colectada aproximadamente una cantidad de $120 \mathrm{~kg}$ de suelo por cada tipo de suelos a una profundidad de $0-20 \mathrm{~cm}$. Estos suelos fueron caracterizados químicamente (Tabla 1) y mineralógicamente (Tabla 2) de acuerdo a las metodologías propuestas por el Instituto Geográfico Agustín Codazzi (2006).

\section{Caracterización química y mineralógica de los suelos}

Como se observa en la tabla 1, las muestras de suelos presentaron $\mathrm{pH}$ con promedio de 7,73 los cuales se consideran suelos ligeramente alcalinos a alcalinos ya que su $\mathrm{pH}$ es mayor a 7,3. Contenidos de materia orgánica muy baja, altos contenidos de fósforo con $65,27 \mathrm{mg} \mathrm{kg}^{-1}$ y contenidos de azufre medios. Las bases intercambiables como el contenido de calcio con promedio de 58,3 se consideran altas y relaciones catiónicas con $\mathrm{Mg}$ y K muy altas. Además, presentan alta capacidad de intercambio catiónico y bajos contenidos de elementos menores que son representativos de estos suelos ligeramente alcalinos.

Tabla 1. Caracterización química de las muestras de suelos en estudio.

\begin{tabular}{|c|c|c|c|c|c|c|c|c|c|c|c|c|c|}
\hline \multirow{2}{*}{ Suelos } & pH & M.O & $\mathbf{S}$ & $\mathbf{P}$ & $\mathrm{Ca}$ & $\mathrm{Mg}$ & $\mathbf{K}$ & $\mathbf{N a}$ & CICE & $\mathbf{C u}$ & $\mathbf{F e}$ & Zn & Mn \\
\hline & $1: 1$ & $\%$ & \multicolumn{2}{|c|}{$--\mathrm{mg} \mathrm{kg}^{-1}$-- } & \multicolumn{5}{|c|}{ 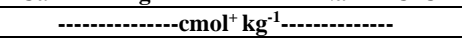 } & \multicolumn{4}{|c|}{ 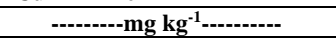 } \\
\hline Moñitos & 7,76 & $\mathbf{0 , 5 1}$ & 20,1 & 65,2 & 87,40 & 1,70 & $\mathbf{0 , 8 3}$ & $\mathbf{0 , 3 0}$ & 90,2 & 0,4 & 0,4 & 0,4 & 1,2 \\
\hline Planeta rica & 7,95 & 1,20 & 20,7 & 40,4 & 42,50 & $\mathbf{0 , 8 0}$ & 0,32 & 0,11 & 43,7 & 0,4 & 9,2 & 0,4 & 0,8 \\
\hline Promedio & 7,73 & 0,97 & 19,77 & 65,27 & 58,30 & 4,17 & 0,48 & 0,28 & 63,20 & 0,40 & 3,60 & 0,40 & 12,40 \\
\hline Mínimo & 7,47 & $\mathbf{0 , 5 1}$ & 18,50 & 40,40 & 42,50 & $\mathbf{0 , 8 0}$ & 0,28 & 0,11 & 43,70 & 0,40 & 0,40 & 0,40 & $\mathbf{0 , 8 0}$ \\
\hline
\end{tabular}

pH: relación suelo-agua 1:1; M.O: Walkley - Black; S: fosfato de calcio monobásico 0,008 mol L-1; P: Bray - II modificado; Ca-Mg-K-Na: Acetato de amonio 1,0 $\mathrm{mol} \mathrm{L}^{-1} \mathrm{pH}$ 7,0; $\mathrm{Al}+\mathrm{H}$ : KCl 1,0 mol L-1. CICe: Suma de bases; $\mathrm{Cu}-\mathrm{Fe}-\mathrm{Zn}$ Mn: Mehlich $^{-1}$ 
En la caracterización mineralógica de arcillas para los suelos alcalinos (Tabla 2), se encontró que los suelos de Moñitos, Planeta rica y Sincelejo, presentan abundancia en arcillas tipo montmorillonitas y presencia de cuarzo, indicando la presencia de silicio en forma de silica amorfa $\mathrm{SiO}_{4}$ en estos suelos. Sin embargo, la presencia de silicio en estos suelos, no implica necesariamente su disponibilidad para planta o en la fase soluble del suelo.

Tabla 2. Caracterización cualitativa y cuantitativa de la mineralogía de los suelos evaluados.

\begin{tabular}{cccc}
\hline SUELOS & Moñitos & Planeta rica & Sincelejo \\
\hline Esmectitas (Montmorillonita) & \multicolumn{3}{c}{ Semicuantitativo* } \\
\cline { 2 - 4 } Intergrados 2:1-2:2 & +++ & ++++ & ++++ \\
Micas & - & - & - \\
Caolinita & ++ & $\operatorname{tr}$ & + \\
Cloritas & $\operatorname{tr}$ & + & $\operatorname{tr}$ \\
Cuarzo & - & - & - \\
Goetita & ++ & + & + \\
Feldespatos & - & + & - \\
Calcita & - & - & - \\
Interestratificados & ++ & - & - \\
Lepidocrocita & $\operatorname{tr}$ & $\operatorname{tr}$ & - \\
\hline
\end{tabular}

* Convenciones: ++++ dominante (>50\%); +++ abundante (30-50\%); ++ común (15-30\%); + presente $(5-15 \%)$; tr trazas $(<5 \%)$.

Para la etapa de invernadero, los $120 \mathrm{~kg}$ de cada uno de tres suelos fueron secados a temperatura ambiente y pasadas por un tamiz de $4 \mathrm{~mm}$ con el fin homogenizarlas $y$ mantener las condiciones edáficas de su procedencia.

En la investigación fue utilizado un diseño completamente al azar con arreglo factorial, $2 \times 3$ (dos cultivos, tres suelos) con 4 repeticiones, para un total de 24 unidades experimentales. En el cual cada unidad experimental (UE) estuvo compuesta por un recipiente plástico de $5 \mathrm{~kg}$ de suelo. Donde cada suelo correspondía a 8 (UE), cuatro fueron sembrados con semillas de maíz de variedad "Pionner" (P-40) y cuatro por frijol utilizando la variedad Caupí LX-CP-M11, colocando en cada unidad 5 semillas por UE según correspondiera al cultivo y los requerimientos nutricionales aplicados para los cultivos de frijol y maíz fueron estimados de acuerdo a la caracterización química realizada a los suelos.

Para el cultivo de maíz se trabajó con un requerimiento equivalente a 200,90 y $150 \mathrm{~kg}$ de nitrógeno $(\mathrm{N})$, fosforo $(\mathrm{P})$ y potasio $(\mathrm{K})$. Adicionándole a cada UE $0,5 \mathrm{~g}$ de $\mathrm{N}$, para el suelo de Moñitos; 0,45g de $\mathrm{N}$ para el suelo de Planeta Rica y 0,45 g de $\mathrm{N}$ para el suelo de Sincelejo y fertilizante utilizado fue la urea. Para fósforo fue necesario aplicar 0,21 $\mathrm{g}$ de P/UE, éste fue aplicado en forma de DAP (Fosfato Diamónico) y de 0,32 g de potasio por UE, aplicado en forma de $\mathrm{KCl}$. Para el cultivo de fríjol se utilizó $0,35 \mathrm{~g}$ de $\mathrm{N}$ en forma de urea, $0,21 \mathrm{~g}$ de $\mathrm{P}$ en forma de DAP y $0,32 \mathrm{~g}$ de $\mathrm{K}$ en forma de $\mathrm{KCl}$ por unidad experimental. En todos los casos, el fósforo se 
aplicó una única vez al momento de la siembra de los cultivos y para $\mathrm{N}$ y $\mathrm{K}$ fueron aplicados en forma fraccionada dos veces, a los 10 y 20 días después de la emergencia de los cultivos respectivamente.

Luego de haber transcurrido 35 días después de la emergencia se realizó la colecta de las plantas de cada tratamiento. Las muestras de tejido vegetal tanto de frijol como de maíz fueron sometidas a secado a una temperatura de $70{ }^{\circ} \mathrm{C}$ en una estufa eléctrica Binder ED-53 durante 72 horas, luego fueron sometidas a molienda por un micromolino IKA MFC S1. Posteriormente, se realizó digestión seca con cada una de las muestras, tomando $0,5 \mathrm{~g}$ de tejido vegetal, se colocaron en una mufla eléctrica, aumentando gradualmente la temperatura hasta $500{ }^{\circ} \mathrm{C}$ la cual fue mantenida por 3 horas. Las cenizas se depositaron en tubos de polietileno y se adicionó $50 \mathrm{~mL}$ de $\mathrm{NaOH}$ al $1 \%$ y la cuantificación se realizó en un equipo de absorción molecular (Embrapa, 2009).

Para determinar los contenidos de silicio disponible, se colectaron submuestras de suelo por cada unidad experimental, posteriormente fueron secadas a temperatura ambiente, trituradas en un molino eléctrico marca Thurm TGL-8394 y tamizadas por 2 mm usando tamices Soilstest ASTM E-11y empacadas en recipientes plásticos herméticamente cerrados para evitar la humedad. Para cuantificar el contenido de Si disponible en cada submuestra de suelo, fueron utilizados cuatro métodos de extracción: extracción con cloruro de calcio $\left(\mathrm{CaCl}_{2}\right)$ 0,01 mol L-1 (método de referencia) (Korndörfer et al., 2004), ácido acético $\left(\mathrm{HC}_{2} \mathrm{H}_{3} \mathrm{O}_{2}\right)$ 0,5 mol L ${ }^{-1}$ (Korndörfer et al., 2004), acetato de amonio $\left(\mathrm{CH}_{3} \mathrm{COONH}_{4}\right)$ $38,5 \mathrm{~g} \mathrm{~L}^{-1}$ (Pereira et al., 2007) y para la extracción con agua modificada con boro que fue el método propuesta por los autores fueron pesados $5 \mathrm{~g}$ de suelo y posteriormente se le agregaron $49 \mathrm{~mL}$ de agua $+1 \mathrm{~mL}$ de
$\mathrm{BaCl}_{2}$ al $10 \%$ y para los tres métodos iniciales se utilizó una relación 5 gramos de suelo con $50 \mathrm{~mL}$ de solución extractora, agitados por 1 hora a $220 \mathrm{rpm}$.

Después de la agitación de cada suelo, se procedió a filtrarlos en papel número 42 y las soluciones filtradas se dejaron en reposo por un periodo de 15 horas. Para la determinación de silicio se tomó una alícuota de $10 \mathrm{~mL}$ del extracto filtrado de cada muestra y colocada en un vaso plástico de $50 \mathrm{~mL}$; al cual se le adicionó $1 \mathrm{~mL}$ de solución sulfomolibdica para la formación del complejo $\beta$ molibdosilicato de color amarillo que ocurre después de 5 minutos a pH 1,4 y 2,0. Una excepción ocurre cuando fue utilizada el acetato de amonio $\left(\mathrm{CH}_{3} \mathrm{COONH}_{4} 38,5 \mathrm{~g} \mathrm{~L}^{-1}\right)$ ya que hubo la necesidad de disminuir el $\mathrm{pH}$ de la solución antes de la aplicación de la solución sulfomolibdica adicionándole $3 \mathrm{~mL}$ de ácido clorhídrico $1 \mathrm{~mol} \mathrm{~L}^{-1}$ en $10 \mathrm{~mL}$ de extracto.

Después de transcurridos 10 minutos de la adición de la solución sulfomolibdica, se adicionaron $2 \mathrm{~mL}$ de ácido tartárico $200 \mathrm{~g} \mathrm{~L}^{-1}$ utilizado para acomplejar el fósforo de la solución. Después de 5 minutos se adicionó 10 $\mathrm{mL}$ de ácido ascórbico $3 \mathrm{~g} \mathrm{~L}^{-1}$ para la cuantificación de silicio por la reducción del complejo $\beta$-molibdosilicato amarrillo a azul de molibdeno (Korndörfer et al., 2004). Finalmente, después de transcurrir 1 hora de reposo se realizó la lectura en un espectrofotómetro Perkim Elmer lambda XLS+ a una longitud de onda de $660 \mathrm{~nm}$.

Los datos de los contenidos de silicio disponible extraído por los diferentes métodos en para cada suelo y los valores cuantificados de silicio en tejidos vegetales de frijol y maíz, fueron sometidos a análisis de variancia y correlación por el software estadístico SAS (Statistical Analysis System) versión 8.0. 


\section{RESULTADOS Y DISCUSIÓN}

\section{Extracción de silicio de los suelos alcalinos}

El método que consiguió extraer los mayores contenidos de silicio disponible fue el ácido acético $0,5 \mathrm{~mol} \mathrm{~L}^{-1}$ (M2) con promedio de $222,85 \mathrm{mg} \mathrm{kg}^{-1}$ en los suelos cultivados con maíz y $197,263 \mathrm{mg} \mathrm{kg}^{-1}$ para suelos cultivados con frijol (Tabla 3). Esto ocurre debido posiblemente a la mayor capacidad de extracción de silicio disponible en estos suelos, permitiendo solubilizar pequeñas cadenas poliméricas que no se encuentran en la fase disponible del suelo. Por lo tanto, este Si es sobreestimado y no podría ser absorbida por la planta (Pereira et al., 2007). Korndörfer et al., (2004), indican que el ácido acético puede solubilizar pequeñas cadenas de $\mathrm{Si}$ polimerizado.

Así mismo, y siguiendo el orden de extracción del $\mathrm{Si}$, el acetato de amonio con 38,5 $\mathrm{g} \mathrm{L}^{-1}$ (M3) fue el segundo método en extracción de este elemento, logrando extraer mayor cantidad que el método de referencia (M1) (Tabla 3). Según Crusciol et al. (2018) el éxito del extractante de acetato de amonio, se debe a la presencia del anión acetato que puede favorecer la desorción del silicato adsorbido, pero también sobreestima el contenido de silicio disponible.

Tabla 3. Contenidos de silicio ( $\mathrm{Si}$ ) disponible extraído con diferentes métodos de extracción y su contenido en cultivos indicadores.

\begin{tabular}{|c|c|c|c|c|c|}
\hline \multirow{3}{*}{ Suelos } & \multicolumn{5}{|c|}{ Cultivo de Maíz } \\
\hline & M1 & M2 & M3 & M4 & Si en Planta \\
\hline & \multicolumn{4}{|c|}{ - } & -.-- $\mathrm{g} \mathrm{kg}^{-1}-\cdots$ \\
\hline Moñitos & 24,195 & 121,045 & 31,144 & 34,454 & 5,027 \\
\hline Planeta rica & 16,621 & 266,180 & 31,142 & 19,558 & 1,349 \\
\hline Sincelejo & 28,158 & 278,858 & 41,365 & 32,411 & 3,586 \\
\hline Promedio & 22,992 & 222,028 & 34,551 & 28,808 & 3,321 \\
\hline Mínimo & 16,621 & 121,045 & 31,142 & 19,558 & 1,349 \\
\hline \multirow[t]{2}{*}{ Máximo } & 28,158 & 278,858 & 41,365 & 34,454 & 5,027 \\
\hline & \multicolumn{5}{|c|}{ Cultivo de Frijol } \\
\hline Moñitos & 26,000 & 99,155 & 34,453 & 34,843 & 0,700 \\
\hline Planeta rica & 15,514 & 262,200 & 30,070 & 25,887 & 0,445 \\
\hline Sincelejo & 26,772 & 230,435 & 38,832 & 34,743 & 0,492 \\
\hline Promedio & 22,762 & 197,263 & 34,452 & 31,824 & 0,546 \\
\hline Mínimo & 15,514 & 99,155 & 30,070 & 25,887 & 0,445 \\
\hline Máximo & 26,772 & 262,200 & 38,832 & 34,843 & 0,700 \\
\hline
\end{tabular}

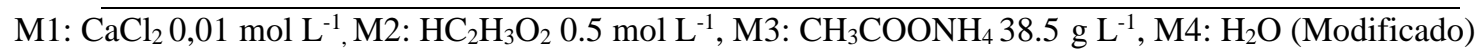

El método de cloruro de calcio $\left(\mathrm{CaCl}_{2}\right) 0,01$ mol L $\mathrm{L}^{-1}$ (M1) fue el método que extrajo los menores contenidos de silicio en el suelo con 22,99 y $22,762 \mathrm{mg} \mathrm{kg}^{-1}$ de Si para maíz y frijol respectivamente, seguido por el método de agua modificada (M4). Así mismo, y de acuerdo con el principio de equilibrio químico del suelo, el Si extraído con $\mathrm{CaCl}_{2}$ diluido puede corresponder más estrechamente a los niveles de Si esperados de las predicciones de solubilidad (Lindsay,
1979; Liang et al., 2015a). Sin embargo, Berthelsen et al. (2001) y Combatt et al. (2017) indican que el agente de extracción más débil después del agua es el $\mathrm{CaCl}_{2}$, que solo extrae la fracción de $\mathrm{Si}$ fácilmente soluble, debido al pH de la solución y a la fuerza iónica que evita la dispersión y facilita la extracción del Si en muestras de suelos. Sin embargo, y de acuerdo con Haynes (2014) el agua ha sido usada en muchos trabajos para estimar el Si soluble 
extraíble. Sin embargo, la extracción con agua diluye en gran medida la fuerza iónica de la solución del suelo, de modo que las cantidades extraídas serán diferentes a las verdaderamente disponibles a las que se encuentran en la solución del suelo. Por otra parte, Miles et al. (2014) y Berthelsen et al. (2005) y Lima et al. (2011) prefieren las soluciones salinas no tamponadas como $\mathrm{CaCl}_{2}\left(0.01 \mathrm{~mol} \mathrm{~L}^{-1}\right)$ ya que tienen una fuerza iónica similar a la fuerza iónica de la solución del suelo

La tendencia de los métodos de extracción encontrados en esta investigación difiere de la reportada por Combatt et al. (2017), quienes mencionan que para suelos alcalinos del departamento de Córdoba, la tendencia en extracción de silicio fue en su orden $\left(\mathrm{HC}_{2} \mathrm{H}_{3} \mathrm{O}_{2}\right.$ 0,5 mol L$\left.{ }^{-1}\right)>$ (Tampón pH 4,0 $\left(\mathrm{HC}_{2} \mathrm{H}_{3} \mathrm{O}_{2}+\mathrm{CH}_{3} \mathrm{COONa}\right)>$ (solución de cloruro de bario) > $\left(\mathrm{CaCl}_{2} \mathrm{~mol} \mathrm{~L} \mathrm{~L}^{-1}\right)>$ $\left(\mathrm{CH}_{3} \mathrm{COONH}_{4} \quad 38,5 \mathrm{~g} \quad \mathrm{~L}^{-1}\right)$, con una extracción de 148,36; 112,85; 24,26; 21,18 y $16,97 \mathrm{mg} \mathrm{kg}^{-1}$ de $\mathrm{Si}$ respectivamente.

Con los resultados obtenidos en la comparación entre los métodos, se puede destacar que los extractantes ácido acético $0,5 \mathrm{~mol} \mathrm{~L}^{-1} \mathrm{y}$ acetato de amonio $38,5 \mathrm{~g} \mathrm{~L}^{-1}$ fueron los que mayor cantidad de silicio consiguieron extraer de los suelos alcalinos y que el cloruro de calcio $0,01 \mathrm{~mol} \mathrm{~L}^{-1}$, fue el que presentó la menor extracción. Además, los resultados de las pruebas estadísticas realizadas indicaron que el método propuesto por los autores, con una solución de cloruro de bario, tiene la misma tendencia en la extracción de silicio disponible en los suelos alcalinos, que el método de referencia $\mathrm{CaCl}_{2}$ 0,01 mol L'${ }^{-1}$.

\section{Correlación entre los métodos de extracción y el Si extraído por las plantas de maíz}

En la figura 1 se observan las correlaciones entre las metodologías de extracción y los contenidos de Si extraídos por el cultivo de maíz. Al analizar la correlación entre el método de referencia (Cloruro de calcio 0,01 mol L ${ }^{-1}$ ) en los suelos alcalinos muestreados (Figura 1a) y los contenidos de silicio extraídos por las plantas, se observa que el coeficiente de correlación (r) fue positivo y significativo $(p<0,05)\left(0,71^{*}\right)$. El Si extraído por el método de ácido acético (Figura 1b) se correlacionó negativa y significativamente $(r=-0,72 *)$ con el $\mathrm{Si}$ extraído por el cultivo de maíz. Así mismo, se observa que, en los suelos alcalinos, para el método de acetato de amonio no se presentó correlación significativa (Figura 1c), finalmente se encontró que hubo correlación positiva entre el método de agua modificada y la extracción de $\mathrm{Si}$ por la especie (Figura 1d), con una correlación altamente significativa $(p<0,01) \quad(\mathrm{r}=$ $0,92 * *)$. 


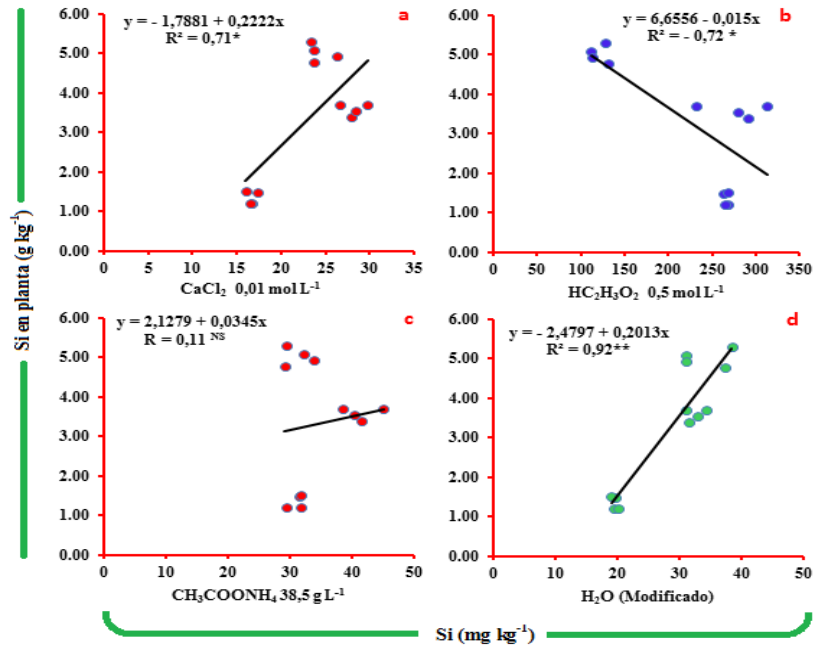

Figura 1. Correlación entre los contenido de $\mathrm{Si}$ extraídos con el cultivo de maíz y los métodos evaluados; a: $\mathrm{CaCl}_{2} 0,01 \mathrm{~mol} \mathrm{~L}^{-1}$, b: $\mathrm{HC}_{2} \mathrm{H}_{3} \mathrm{O}_{2} 0,5 \mathrm{~mol} \mathrm{~L}^{-1}$, c: $\mathrm{CH}_{3} \mathrm{COONH}_{4} 38,5 \mathrm{~g} \mathrm{~L}^{-1}, \mathbf{d}: \mathrm{H}_{2} \mathrm{O}$ (Modificado por autores).

De acuerdo con estos resultados se teoriza que el método de referencia y el método de agua modificada constituyen las metodologías más convenientes para la extracción de Si por parte de del cultivo de maíz en suelos alcalinos. Esta extracción del Si disponible por estos métodos se puede explicar por las características fisicoquímicas que influyen en estos extractores, como son los cationes índices $\mathrm{Ca}^{2+} \mathrm{y} \mathrm{Ba}^{2+}$ que pueden desplazar el Si más fácilmente extraíble de estos suelos alcalinos. Al respecto, Combatt et al. (2017), encontraron una correlación positiva entre el método de referencia y el método de agua modificada (cloruro de bario), para la extracción de $\mathrm{Si}$ en suelos alcalinos del departamento de Córdoba $\left(r=0,93^{*}\right)$. Los mismos autores describen que esta asociación se puede explicar por el poder de extracción de estas soluciones que está en función de las interacciones catión índice y el elemento a desplazar (Si) y químicamente la reacciones que ocurren logran un equilibrio similar para la extracción del silicio disponible del suelo.

Por otra parte, el método de ácido acético 0,5 mol L ${ }^{-1}$ (Figura $1 b$ ), presenta una pendiente negativa evidenciando una desfavorable correlación y una baja homogeneidad entre sus puntos, lo cual indica que, a valores altos de silicio extraído por las plantas de maíz, le corresponden valores bajos de Si extraído del suelo por el método de ácido acético. Además, este extractor es una solución acida, que posiblemente origine la sobreestimación de los contenidos de silicio extraídos, debido a la gran cantidad de iones hidronios que solubilizan el silicio que no están disponibles para las plantas. Este trabajo contrasta con el reporte de Crusciol et al. (2018), quienes denotan que el ácido acético con el método convencional no sobreestimó la disponibilidad de $\mathrm{Si}$ en el suelo y proporcionó altos coeficientes de correlación entre el contenido de Si soluble en el suelo y las cantidades de $\mathrm{Si}$ absorbidas por el cultivo de caña de azúcar. Así mismo, estos resultados difieren de los estudios de correlación que evaluó Filho et al. (2000), quienes determinaron que el Si extraído con ácido acético 0,5 M fue más adecuado para valorar el Si extraíble, seguido de extracción con ácido cítrico al $1 \%$. Así mismo, estos investigadores encontraron correlaciones con el porcentaje de Si en cáscara y panícula de $\operatorname{arroz}\left(\mathrm{r}=0.899^{* *}, \mathrm{r}=0.768^{* *}\right.$ y $\mathrm{r}=$ $\left.0.839^{* *}, \mathrm{r}=0.774^{* *}\right)$.

Finalmente, la ausencia de significancia en la correlación de la extracción de Si por parte 
del método de acetato de amonio $38,5 \mathrm{~g} \mathrm{~L}^{-1}$ pudo deberse al efecto de una extracción de Si heterogénea y no uniforme, ocasionado por las características de los compuestos de silicio en estos suelos, que pueden ser extraídos por la solución acida que origina el acetato de amonio (Crusciol et al. (2018).

\section{Correlación entre los métodos de extracción y el Si extraído por las plantas de frijol.}

Para el análisis de correlación de los contenidos de silicio disponible en suelos extraído por diferentes métodos y los contenidos de silicio en el cultivo de frijol (Figura 2), se encontró que no hubo correlaciones altamente significativas para los extractantes, con excepción del método de ácido acético $0,5 \mathrm{~mol} \mathrm{~L}^{-1}$ el cual presentó correlación significativa $(p<0,05)$, pero su línea de tendencia mostró un comportamiento inversamente proporcional $\left(\mathrm{R}^{2}=-0,71 *\right)$ a lo extraído en el suelo con lo acumulado por el cultivo(Figura 2b).
El método de ácido acético $0,5 \mathrm{~mol} \mathrm{~L}^{-1}$ sobrestima los contenidos de silicio disponible para la planta en comparación con la capacidad de extracción de soluciones ligeramente neutras, debido a que este es capaz de solubilizar el $\mathrm{Si}$ de compuestos presenten en el suelo que no son solubles para la planta, tal como formas polimerizadas (ácido polisilícico) (Crusciol et al., 2018). De acuerdo con Liang et al. (2015b) a pH entre 2 y 9, el Si del suelo se encuentra presente principalmente como ácido monosilícico $\left(\mathrm{H}_{4} \mathrm{SiO}_{4}\right)$ que es disuelto y cuantificado como disponible. Por lo tanto, en su cuantificación se puede presentar una baja y negativa correlación cuando se utiliza el ácido acético como extractor (Pereira et al., 2007). Pero, cuando son utilizados otros métodos, estos no logran solubilizar todos los compuestos que contienen silicio, como ocurre con el cloruro de calcio $0,01 \mathrm{~mol} \mathrm{~L}^{-1}$ y el método propuesto. Resultados semejantes fueron obtenidos por Berthelsen et al. (2002) y Sumida (2002), mostrando la eficiencia de las soluciones ácidas, comparadas con soluciones neutras.

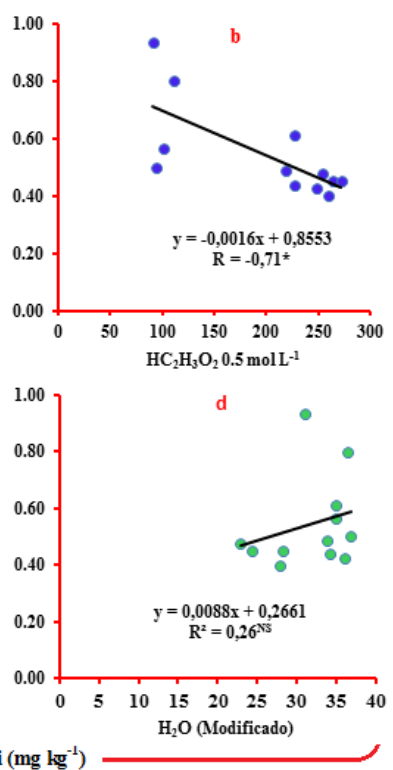

Figura 2. Correlación entre los contenido de $\mathrm{Si}$ extraídos con el cultivo de frijol y los métodos evaluados; a: $\mathrm{CaCl}_{2}$ 0,01 mol L-1, b: $\mathrm{HC}_{2} \mathrm{H}_{3} \mathrm{O}_{2}$ 0,5 mol L-1 $, \mathbf{c}: \mathrm{CH}_{3} \mathrm{COONH}_{4} 38,5 \mathrm{~g} \mathrm{~L}^{-1}, \mathbf{d}: \mathrm{H}_{2} \mathrm{O}$ (Modificado). 
En general, los métodos $\mathrm{CaCl}_{2}$ y agua modificada, no presentaron correlacionaron significativas con el $\mathrm{Si}$ extraído por las plantas de frijol en los suelos alcalinos evaluados. El bajo nivel de extracción de Si por parte de la planta y la falta de uniformidad en la extracción de Si por los métodos mencionados, pueden explicar esta ausencia de significancia (Crusciol et al., 2018; Inocencio et al., 2010). De acuerdo con Gutzer et al. (2012) la absorción por la planta de este elemento, se ve afectada por los contenidos de silicio disponible en la solución del suelo, ya que para que ocurra una buena absorción debe existir suficiente silicio disponible para evidenciar respuestas del cultivo. Sin embargo, este no es un criterio absoluto para explicar los niveles de $\mathrm{Si}$ en las plantas sembradas en los suelos evaluados.

Estos resultados también concuerdan con De Albuquerque et al. (2011), quienes aplicaron diferentes dosis de silicio en cultivos de frijol y no encontraron repuestas a la aplicación de este elemento. En el mismo sentido, Inocencio et al. (2010) y Gutzer et al. (2012) explican que el cultivo de frijol no se considera acumulador de Si. Por lo tanto, no se observan altos contenidos de silicio extraído por este cultivo. Sin embargo, Crusciol et al. (2018) reportan que hubo una correlación positiva y significativa entre el contenido de Si del suelo y las cantidades de $\mathrm{Si}$ acumuladas en el cultivo caña de azúcar en todas las clases texturales de suelo evaluados, con correlaciones que oscilaron entre 0,796 y $0,946(p<0,001)$. Hodson et al. (2005) consideran que la concentración de $\mathrm{Si}$ en las plantas depende principalmente de la posición filogenética de la planta más que de su entorno, es decir, la concentración de $\mathrm{Si}$ en el suelo y $\mathrm{pH}$ del suelo.

De acuerdo con Narayanaswamy y Prakash (2009) la variación encontrada en los niveles de Si extraídos en los suelos cultivados y el silicio acumulado para los cultivos maíz y para frijol, fueron diferentes $y$ probablemente explicado por la naturaleza de los cultivos indicadores y por el poder de extracción de los extractores, el pH de la solución de extracción, el período de agitación, la relación suelo / solución, así como la naturaleza de los diferentes extractores utilizados.

\section{CONCLUSIÓNES}

El método más eficaz para extraer silicio en de suelos alcalinos para el cultivo de frijol y maíz fue el método de ácido acético 0,5 mol $\mathrm{L}^{-1}$ y el que menos extrajo fue el cloruro de calcio $0,01 \mathrm{~mol} \mathrm{~L}^{-1}$.

El método que presentó la mayor correlación con el cultivo de maíz fue el método de agua modificada propuesto por los autores, seguido por el método de cloruro de calcio $0,01 \mathrm{~mol} \mathrm{~L}^{-1}$ y para el cultivo de frijol caupi no se presentó correlación con los diferentes métodos.

En las dos especies indicadoras, se presentó extracción de silicio en magnitudes estadísticamente diferentes, situación que se asoció a las diferencias genéticas entre estos cultivos y al contenido de $\mathrm{Si}$ disponible en cada suelo.

De los extractantes utilizados el método de referencia y el método propuesto por los autores pueden ser una alternativa en la estimación de silicio en suelos alcalinos cuando se establecen gramíneas como el maíz.

Es necesario seguir adelantando estudio en metodologías analíticas para la extracción de silicio, ya que son pocos los laboratorios de suelos en Colombia que ofrecen este servicio $\mathrm{y}$ hay muchas empresas ofreciendo el servicio de venta de silicio para un gran número de cultivos. 


\section{REFERENCIAS}

Babu, T., Tubana, B., Paye, W., Kanke, Y. y Datnoff, L. (2016). Establishing soil silicon test procedure and critical silicon level for rice in Louisiana Soils. Communications in Soil Science and Plant Analysis, 47(12), 1578-1597. doi: 10.1080/00103624.2016.1194996

Berthelsen, S. y Korndörfer, G. H. (2005). Methods for Silicon analysis in soil, plant and fertilizers. In: Proceedings of the third International Conference on Silicon in Agriculture. Uberlândia. Proceedings.... Uberlândia: Universidade Federal de Uberlândia, 85-90.

Berthelsen, S., Noble, A. D. y Garside, A. L. (2001). Silicon research down under: past, present, and future. In Studies in Plant Science, (8), 241-255). Elsevier.

Berthelsen, S., Noble, A., Kingston, G., Hurney, A. y Rudd, A. (2002). Effect of Casilicate amendments on soil chemical properties under a sugarcane cropping system. In Silicon in agriculture conference, (2), 57).

Combatt C., E., Palacio B., D., y Mercado L. J. (2017). Extractores químicos en la determinación de silicio disponible en suelos ácidos, neutros y alcalinos. Rev. U.D.C.A Act. \& Div. Cient, 20(2), 329-339.

Crooks, R. y Prentice, P. (2017). Extensive investigation into field based responses to a silica fertiliser. Silicon, 9(2), 301-304.

Crusciol, C. A. C., De Arruda, D. P., Fernandes, A. M., Antonangelo, J. A., Alleoni, L. R. F., do Nascimento, C. A. C., ... y McCray, J. M. (2018). Methods and extractants to evaluate silicon availability for sugarcane. Scientific reports, 8(1), 916. doi:10.1038/s41598-018-19240-1
De Albuquerque-Lima, M., de Castro, V. F., Vidal, J. B., \& Enéas-Filho, J. (2011). Silicon application on plants of maize and cowpea under salt stress. Revista Ciência Agronômica, 42(2), 398.

Empresa Brasileira de Pesquisa Agropecuária - EMBRAPA. 2009.Centro Nacional de Pesquisa de Solos. Manual de métodos de análise de solos. 2.ed. Rio de Janeiro, EMBRAPA-CNPS,. 212p.

Filho, B., M.P; Zinder, G.H; Prabhu, A.S; Datnoff, L.E; Kornörfer, G.H. 2000. Importancia do silicio para a cultura do arroz. Uma revisão de literatura. Potafos. Encarte Técnico. Informaciõnes AgronÕmicas No 89.

Guntzer, F., Keller, C., y Meunier, J. D. (2012). Benefits of plant silicon for crops: a review. Agronomy for Sustainable Development, 32(1), 201-213. doi: 10.1007/s13593-011-0039-8

Haynes, R. J. (2014). A contemporary overview of silicon availability in agricultural soils. Journal of Plant Nutrition and Soil Science, 177(6), 831-844. doi: 10.1002/jpln.201400202

Haynes, R. J. (2017). The nature of biogenic $\mathrm{Si}$ and its potential role in $\mathrm{Si}$ supply in agricultural soils. Agriculture, Ecosystems \& Environment, 245, 100-111. doi: 10.1016/j.agee.2017.04.021

Hodson, M. J., White, P. J., Mead, A. y Broadley, M. R. (2005). Phylogenetic variation in the silicon composition of plants. Annals of botany, 96(6), 1027-1046. doi: 10.1093/aob/mci255

Huang, C. H., Roberts, P. D. y Datnoff, L. E. (2011). Silicon suppresses Fusarium crown and root rot of tomato. Journal of 
Phytopathology, 159(7-8), 546-554. doi: 10.1111/j.1439-0434.2011.01803.x

Inocêncio, M. F., Gutierrez, R. S. y Novelino, J. O. (2010). Avaliação de métodos de extração de silício e fósforo em amostras de solos. Revista Caatinga, 23(4), 75-83.

Instituto Geográfico Agustín Codazzi IGAC (2006). Métodos analíticos del laboratorio de suelos. VI Edición. Bogotá, Subdirección de Agrología, 499 p.

Korndörfer, G. H., Pereira, H. S. y Nolla, A. (2004). Análise de silício: solo, planta e fertilizante. 2 ed. Uberlândia: Instituto de Ciências Agrárias, Universidade Federal de Uberlândia. 34 p.

Liang Y., Nikolic M., Bélanger R., Gong H. y Song A. (2015b) Silicon Biogeochemistry and Bioavailability in Soil. In: Silicon in Agriculture (45-68). Springer, Dordrecht. doi: 10.1007/978-94-017-9978-2_3

Liang, Y., Nikolic, M., Bélanger, R., Gong, H. y Song, A. (2015a). Silicon in agriculture. Springer, Dordrecht. doi: 10.1007/978-94-017-9978-2_2

Lima, M,A., Freitas, V., Vidal, J,V ., Filho, E,J. 2011. Silicon application on plants of maize and cowpea under salt stress, Revista Ciência Agronômica, 42(2): 398-403.

Lindsay, L. W. (1979). Chemical equilibria in soils. Chichester, UK: John Wiley \& Sons.

Ma, J. F. y Takahashi, E. (2002). Soil, fertilizer, and plant silicon research in Japan. 11(8).

Meunier, J. D., Sandhya, K., Prakash, N. B., Borschneck, D. y Dussouillez, P. (2018). pH as a proxy for estimating plant-available $\mathrm{Si}$ ? A case study in rice fields in Karnataka
(South India). Plant and soil, 432(1-2), 143155. doi: 10.1007/s11104-018-3758-7

Miles, N., Manson, A. D., Rhodes, R., van Antwerpen, R. y Weigel, A. (2014). Extractable silicon in soils of the South African Sugar industry and relationships with crop uptake. Communications in soil science and plant analysis, 45(22), 29492958. doi: 10.1080/00103624.2014.956881

Narayanaswamy, C. y Prakash, N. B. (2009). Calibration and categorization of plant available silicon in rice soils of South India. Journal of plant nutrition, 32(8), 1237-1254. doi: 0.1080/01904160903005970

Patil, A. A., Durgude, A. G., Pharande, A. L., Kadlag, A. D. y Nimbalkar, C. A. (2017). Periodical status of plant available silicon as influenced by silicon extractants and levels of silicon in alkaline soils. IJCS, 5(6), 166173.

Pereira, H. S., Barbosa, N. C., Carneiro, M. A. C. y Korndörfer, G. H. (2007). Avaliação de fontes e de extratores de silício no solo. Pesquisa Agropecuária Brasileira, 42(2), 239-247.

Sandim, S,A., Aparecida, R,A., Ortiz, D,L. y Savi, M,A. (2010). Doses de silício na produtividade do milho (Zea mays L.) híbrido simples na região de Cam o GrandeMS. Cultivando o saber, Cascavel,3(1), 171-178.

Sauer, D., Saccone, L., Conley, D. J., Herrmann, L. y Sommer, M. (2006). Review of methodologies for extracting plantavailable and amorphous Si from soils and aquatic sediments. Biogeochemistry, 80(1), 89-108. doi: 10.1007/s10533-005-5879-3

Sumida, H. (2002). Plant-available silicon in paddy soils. In Proceedings of the Second 
Silicon in Agriculture Conference, 22-26 August 2002, Tsuruoka, Yamagata, Japan (pp. 43-49). Japanese Society of Soil Science and Plant Nutrition.

Tubana, B. S., Babu, T. y Datnoff, L. E. (2016). A review of silicon in soils and plants and its role in US agriculture: history and future perspectives. Soil Science, 181(9/10), 393-411. doi: $10.1097 /$ SS.0000000000000179 